\title{
A GPC3-specific aptamer-mediated magnetic resonance probe for hepatocellular carcinoma
}

This article was published in the following Dove Press journal: International Journal of Nanomedicine

\author{
Menglong Zhao' \\ Zhuang $\operatorname{Liu}^{2,3}$ \\ Lili Dong 4,5 \\ Hongxin Zhou ${ }^{4,5}$ \\ Shuohui Yang 6 \\ Weizhong $\mathrm{Wu}^{4,5}$ \\ Jiang Lin'
}

'Department of Radiology, Zhongshan Hospital, Fudan University, and Shanghai Institute of Medical Imaging, Shanghai, People's Republic of China; ${ }^{2}$ Department of Radiology, Fudan University Shanghai Cancer Center, Shanghai, People's Republic of China; ${ }^{3}$ Department of Oncology, Shanghai Medical College, Fudan University, Shanghai, People's Republic of China; ${ }^{4}$ Liver Cancer Institute, Zhongshan Hospital, Fudan University, Shanghai, People's Republic of China; ${ }^{5} \mathrm{Key}$ Laboratory of Carcinogenesis and Cancer Invasion, Ministry of Education, Shanghai, People's Republic of China; ${ }^{6}$ Department of Radiology, Shuguang Hospital, Shanghai University of Traditional Chinese Medicine, Shanghai, People's Republic of China
Correspondence: Jiang Lin Department of Radiology, Zhongshan Hospital, Fudan University, I80 Fenglin Road, Shanghai 200032, People's Republic of China

Tel +862164041990

Fax +86 2I 64038472

Email lin.jiang@zs-hospital.sh.cn
Purpose: To construct and test a hepatocellular carcinoma (HCC)-targeted magnetic resonance probe based on a glypican-3 (GPC3)-specific aptamer (AP613-1) with ultrasmall superparamagnetic iron oxide (USPIO).

Methods: Oleic acid-coated USPIO nanoparticles were modified with amino polyethylene glycol on the surface. Amino groups of the USPIO nanoparticles were reacted with the carboxyl group of 5' carboxyl-modified AP613-1, forming an aptamer-mediated USPIO (Apt-USPIO) probe. The material characterization of this probe including transmission electron microscopy (TEM), zeta potential, dynamic laser scattering, and magnetic behavior was carried out. The targeting efficiency and magnetic resonance imaging (MRI) performance of Apt-USPIO were evaluated both in vitro and in vivo with USPIO alone as a control. The cytotoxicity and biocompatibility of Apt-USPIO and USPIO were analyzed by cell counting kit- 8 tests in vitro and animal experiments in vivo.

Results: TEM imaging revealed that the Apt-USPIO nanoparticles were spherical in shape and well dispersed. Specific uptake of Apt-USPIO in Huh-7 cells could be observed using the Prussian blue staining test; however, no uptake of USPIO could be found. In vitro phantom $\mathrm{T}_{2}$-weighted MRI showed a significant decrease of the signal intensity in Apt-USPIO-incubated Huh-7 cells compared to USPIO-incubated Huh-7 cells. In vivo $\mathrm{T}_{2}$-weighted MRI showed significantly negative enhancement in the Huh-7 tumors enhanced with Apt-USPIO, whereas no enhancement was found with USPIO alone. Excellent biocompatibility of Apt-USPIO and USPIO was also demonstrated.

Conclusion: In this study, a molecular MRI probe which was highly specific to GPC3 on HCC was successfully prepared. Our results validated the targeted imaging effect of this Apt-USPIO probe in vivo for GPC3-expressing HCCs in xenograft mice.

Keywords: tumor-targeted imaging, carcinoma, hepatocellular, glypican-3, MRI, aptamer, ultrasmall superparamagnetic iron oxide

\section{Introduction}

Hepatocellular carcinoma (HCC) is the most common primary malignancy of the liver and is one of the leading causes of cancer-related deaths worldwide. ${ }^{1,2}$ Despite efforts to improve prognosis, the overall survival is still dismal. Therefore, detection of HCC at an early stage is paramount for an optimal outcome.

Magnetic resonance imaging (MRI) is a well-established imaging technique widely used for diagnosing HCC. Extracellular or combined extracellular and hepatobiliary gadolinium-based contrast agents are generally used in MRI to detect and characterize liver lesions. ${ }^{3}$ However, they do not specifically target HCC itself, and their diagnostic performance can be impaired by diffuse liver diseases. ${ }^{4}$ 
Application of a contrast agent with specific targeting functionality to $\mathrm{HCC}$ may greatly enhance its early detection. Aptamer is a single-stranded oligonucleotide with a unique tertiary structure that can bind to a wide range of targets with high affinity and specificity. ${ }^{5,6} \mathrm{In}$ contrast to antibodies, it possesses major advantages including small molecular weight, lack of immunogenicity, and stability. These characteristics make it a promising alternative to or even a replacement of an antibody in cancer-targeted imaging. 5,7

Currently, $\alpha$-fetoprotein (AFP) is the most frequently used biomarker for HCCs, but with limited sensitivity in small or early tumors. Glypican-3 (GPC3), which has been investigated extensively in recent years, is a heparan sulfate proteoglycan highly expressed in the plasma membrane of most HCC cells, but not in normal liver tissue or benign hepatocellular nodules. ${ }^{8-11}$ It was considered that GPC3 would be superior to AFP in the detection of small HCCs, and could serve as a potential biomarker for early HCCs. ${ }^{12,13}$ In one recent study, aptamers (AP613-1) with specific affinity to GPC3-positive HCC were screened, and near-infrared fluorescence-labeled aptamers were successfully tested as a probe to image the subcutaneous HCC in xenograft models. ${ }^{14}$ However, due to the poor penetration of fluorescent signals from deep tissues, low spatial resolution, and fluorescence quenching effects, these fluorescence probes are of limited value in clinical applications.

Ultrasmall superparamagnetic iron oxide (USPIO) has shown promise as a non-gadolinium magnetic resonance (MR) contrast agent with small molecular weight, strong magnetic moment, and excellent biocompatibility. ${ }^{15,16}$ After surface modification, USPIO could be coupled with different ligands including monoclonal antibodies, peptides, and aptamers which are specific for certain tumor antigens for improved tumor detection and characterization. In an in vitro experiment, a molecular MR probe for the detection of HCC cells was produced by conjugating USPIO with a GPC3-targeted monoclonal antibody. ${ }^{17}$ Recently, studies have shown the efficacy of aptamer-iron oxide probes for in vivo tumor-targeted MRI. ${ }^{18,19}$ However, to the best of our knowledge, an aptamer-based MR probe specific for HCC has not been reported yet.

The objective of this study was to develop a HCC-targeted USPIO probe (Apt-USPIO) using a GPC3-specific aptamer (AP613-1) that binds to GPC3 on the surface of HCC cells. The targeting efficiency of this novel Apt-USPIO nanoparticle was evaluated on $\mathrm{T}_{2}$-weighted MRI both in vitro and in vivo in a tumor xenograft.

\section{Methods}

\section{Materials and reagents}

Phosphate buffer solution (PBS) was purchased from Shanghai Double-Helix Biotech Co. Ltd. Trypsin (0.25\%)ethylenediamine tetraacetic acid (EDTA), fetal bovine serum (FBS), Dulbecco's Modified Eagle's Medium (DMEM), and penicillin-streptomycin were purchased from Thermo Fisher Scientific (Waltham, MA, USA). Cell counting kit-8 (CCK-8) was obtained from Dojindo Molecular Technologies (Rockville, MD, USA). Other reagents and solvents were of analytical grade and prepared with deionized water.

\section{Construction, characterization, and verification of Apt-USPIO nanoparticles}

Oleic acid (OA)-coated USPIO was modified with 1,2-distearoyl-sn-glycero-3-phosphoethanolamine-N[amino(polyethylene glycol)-2000] (DSPE-PEG ${ }_{2000}-\mathrm{NH}_{2}$; PS2-0-20K; Shanghai Ponsure Biotech Inc., Shanghai, People's Republic of China). Briefly, DSPE-PEG ${ }_{2000}-\mathrm{NH}_{2}$ $(0.15 \mathrm{~g})$ was dissolved in $5 \mathrm{~mL}$ deionized water and then mixed with $0.615 \mathrm{~mL}$ of chloroform solution containing OA-coated USPIO ${ }^{20}$ (Fe concentration: $7.25 \mathrm{mg} / \mathrm{mL}$, So-Fe Biomedicine Co., Ltd., Shanghai, People's Republic of China). They were reacted by ultrasonic dispersion in $55^{\circ} \mathrm{C}$ water for about $75 \mathrm{~min}$. Then, the mixture was stirred at $70^{\circ} \mathrm{C}$ in a water bath for 10 min to remove any organic solvent. The modified USPIO nanoparticle was collected and resuspended in PBS.

Subsequently, $2.9 \mathrm{nmol} \mathrm{5'}$ carboxyl-modified aptamer (AP613-1, 5'-COOH-TAACGCTGACCTTAGCTGCATG GCTTTACATGTTCCA-3'; Thermo Fisher Scientific) was blended with $630 \mu \mathrm{L}$ USPIO (Fe concentration: $1 \mathrm{mg} / \mathrm{mL}$ ) at a 3:1 molar ratio. The mixture was then shaken for $2 \mathrm{~h}$ at room temperature followed by activation with N-hydroxysuccinimide/1-ethyl-3-(3-dimethylaminopropyl) carbodiimide hydrochloride (10 mM; Sigma-Aldrich, St Louis, MO, USA). The products (Apt-USPIO nanoparticles) were subsequently separated by centrifugal ultrafiltration $(5,000 \mathrm{rpm}, 30 \mathrm{~min})$ with a $10 \mathrm{kDa}$ cut-off molecular weight membrane, washed twice with PBS, and resuspended for further use. The concentration of unreacted singlestranded oligonucleotide in the supernatant was measured by a NanoDrop DS-11 Spectrophotometer (DeNovix Inc., Wilmington, DE, USA). To verify this conjugation, electrophoresis (DYCP-31DN electrophoresis apparatus; Beijing Liuyi Biotechnology Co., Ltd.) was done, in which the AP613-1, USPIO, and conjugated Apt-USPIO were loaded 
onto each well of $0.5 \%$ agarose gel with electrophoresis buffer ( $0.5 \times$ Tris-borate-EDTA buffer; Sigma-Aldrich), and run at $110 \mathrm{~V}$ for about $30 \mathrm{~min}$.

Transmission electron microscopy (TEM; JEM 2100; JEOL, Tokyo, Japan) of Apt-USPIO was performed at $80 \mathrm{kV}$ to analyze the core size, morphology, and dispersion quality. In brief, samples prepared for TEM measurement were dropped onto a copper grid, dried in an electronic drying cabinet at $25^{\circ} \mathrm{C}$, and then loaded into the microscope for imaging. To measure the hydrodynamic diameters and surface charges of the USPIO and Apt-USPIO, dynamic laser scattering and zeta potential were analyzed using a Malvern laser granulometer (Zetasizer Nano ZSP; Malvern Instruments, Malvern, UK) at $25^{\circ} \mathrm{C}$. Magnetic properties of the Apt-USPIO were checked by measuring the $\mathrm{T}_{1}$ and $\mathrm{T}_{2}$ relaxation times using a Bruker Mq60 nuclear MR analyzer (Bruker, Billerica, MA, USA) at $37^{\circ} \mathrm{C}$.

\section{Cell culture}

Huh-7 cell line, a human HCC cell line with high GPC3 expression, and L-02, a normal human hepatic cell line without GPC3 expression, were purchased from Shanghai Cell Bank (Chinese Academy of Sciences, Shanghai, People's Republic of China). They were grown in DMEM supplemented with $10 \%$ FBS and $1 \%$ penicillin-streptomycin at $37^{\circ} \mathrm{C}$ in a $5 \% \mathrm{CO}_{2}$ atmosphere.

\section{Cytotoxicity of nanoparticles}

Huh-7 cells and L-02 cells were cultured in 96-well plates (Corning Incorporated, Corning, NY, USA) with $0.1 \mathrm{~mL}$ DMEM at a density of $5 \times 10^{3}$ cells per well for $12 \mathrm{~h}$. The cells were incubated with varied concentrations $(0,12.5,25$, 50,100 , and $200 \mu \mathrm{g} / \mathrm{mL}$ ) of USPIO and Apt-USPIO at $37^{\circ} \mathrm{C}$ for additional 24 and $48 \mathrm{~h}$. The standard CCK-8 assay was performed to determine the cell viabilities according to the protocol from the manufacturer. In brief, $20 \mu \mathrm{L}$ of CCK-8 reagent was added to each well, and the solutions were incubated for $4 \mathrm{~h}$ before absorption. Cell viability was then determined using a microplate reader at $450 \mathrm{~nm}$.

\section{Immunofluorescence staining of GPC3}

Immunofluorescence staining based on an anti-GPC3 antibody (mouse anti-human; $10 \mu \mathrm{g} / \mathrm{L}$; Abcam, Cambridge, UK) was performed on the Huh-7 and L-02 cell lines with a standard protocol to verify the positive expression of GPC3 in Huh-7 cell line.

\section{Intracellular uptake experiments}

The Prussian blue staining technique was used to determine the Apt-USPIO uptake by Huh-7 cells. Huh-7 cells (or L-02 cells, as a negative cell control) were seeded into six-well plates at a density of $1 \times 10^{5}$ cells/well for $12 \mathrm{~h}$ at $37^{\circ} \mathrm{C}$. Subsequently, the cells were incubated with $200 \mu \mathrm{g} / \mathrm{mL}$ Apt-USPIO for $12 \mathrm{~h}$, washed with PBS three times, and fixed with $4 \%$ formaldehyde for $20 \mathrm{~min}$. The cells were stained with a mixture of $2.5 \%$ potassium ferrocyanide and $2.5 \%$ hydrochloric acid for $20 \mathrm{~min}$, and then washed with deionized water for $5 \mathrm{~min}$ before observation. In addition, USPIO was also used as a control.

\section{In vitro phantom $\mathrm{MRI}$ analysis}

For this analysis, $2.5 \times 10^{6} \mathrm{Huh}-7$ cells (or L-02 cells, as a negative cell control) were incubated with USPIO or AptUSPIO at concentrations of $0,30,60,120$, and $240 \mu \mathrm{g} / \mathrm{mL}$ for $3 \mathrm{~h}$, and collected after being washed with PBS three times. The cells were mixed with $1 \mathrm{~mL}$ of $2 \%$ agar $\left(50^{\circ} \mathrm{C}\right)$ and poured into $1 \mathrm{~mL}$ tubes. The suspensions were blended carefully to maintain homogeneity without air bubbles, and subsequently cooled down to room temperature for MR scanning. MRI of the test tubes was performed on a 1.5 T MR scanner (MAGNETOM Aera; Siemens Healthcare, Erlangen, Germany) with a 12-channel head coil. To avoid susceptibility artifacts caused by air interface in the scans, all the tubes were placed in a water-containing plastic case at room temperature. MRI was acquired using an axial, $\mathrm{T}_{2}$-weighted turbo spin-echo (TSE) sequence (repetition time $[\mathrm{TR}]=4,000$ $\mathrm{ms}$, echo time $[\mathrm{TE}]=74 \mathrm{~ms}$, number of excitations $[\mathrm{NEX}]=$ 2 , field of view $[\mathrm{FOV}]=100 \times 100 \mathrm{~mm}$, slice thickness $=$ $2.0 \mathrm{~mm}$, matrix size $=320 \times 320$, and flip angle $=150^{\circ}$ ). Circular regions of interest (ROIs) covering the tube cross section were placed on $\mathrm{T}_{2}$-weighted images, and the signal intensities were measured automatically by a workstation (Syngo Multimodality Workplace; Siemens healthcare, Erlangen, Germany). The eventual signal intensity of each sample was determined by the mean value of all the measured signal intensities of the test tube.

\section{Xenograft model}

This experiment was approved by the Institutional Ethics and Animal Care Committee of Zhongshan Hospital, Fudan University, and all procedures involving animals were conducted in accordance with the laboratory animal care and use guidelines adopted by the National Institutes of Health (Shanghai, China). Twelve male athymic Balb/C (Balb/C-nu) 
mice ( 4 weeks old) were purchased from Shanghai SLAC Laboratory Animal Co., Ltd. and were inoculated subcutaneously with $5 \times 10^{6}$ tumor cells into the right lower flank close to hind limbs. The tumors were allowed to grow for about 3 weeks to reach $1.0-1.5 \mathrm{~cm}$ in diameter before MRI.

\section{In vivo $M R$ acquisition and image analysis}

MRI was performed on the $1.5 \mathrm{~T}$ MR scanner described earlier, equipped with a 16-channel wrist coil. Firstly, the nude mice were randomly divided into experimental and control groups with six mice per group. They were anesthetized with an intraperitoneal injection of pentobarbital sodium solution at a dose of $80 \mathrm{mg} / \mathrm{kg}$ before MRI. Each mouse of the experimental group was injected with $200 \mu \mathrm{L}$ physiological saline containing Apt-USPIO (Fe concentration: $1 \mathrm{mg} / \mathrm{mL}$ ) via a tail vein, whereas each mouse in the control group received the same volume of USPIO (Fe concentration: $1 \mathrm{mg} / \mathrm{mL}$ ) as a control. Axial and coronal TSE $\mathrm{T}_{1}$-weighted and $\mathrm{T}_{2}$-weighted images through the tumors were obtained at preinjection and at 1,2 , and $4 \mathrm{~h}$ postinjection of the Apt-USPIO and USPIO. The $\mathrm{T}_{1}$-weighted TSE sequence parameters were set as follows: $\mathrm{TR}=400 \mathrm{~ms}, \mathrm{TE}=12 \mathrm{~ms}, \mathrm{NEX}=2, \mathrm{FOV}=$ $100 \times 100 \mathrm{~mm}$, slice thickness $=2.0 \mathrm{~mm}$, matrix size $=192 \times$ 192, and flip angle $=90^{\circ}$. The $\mathrm{T}_{2}$-weighted imaging sequence parameters were as follows: $\mathrm{TR}=4,000 \mathrm{~ms}, \mathrm{TE}=74 \mathrm{~ms}$, $\mathrm{NEX}=2, \mathrm{FOV}=100 \times 100 \mathrm{~mm}$, slice thickness $=2.0 \mathrm{~mm}$, matrix size $=320 \times 280$, and flip angle $=150^{\circ}$. On coronal $\mathrm{T}_{2}$-weighted images, ROIs were drawn to outline the solid part of the tumor on each slice. The signal intensity from each ROI was measured automatically. The signal intensity for a mass was presented as the average value from all the measured ROIs. According to a previous report, ${ }^{18}$ signal intensities of the back muscle adjacent to the tumor were also measured, and the relative signal enhancement (RSE) of the tumor was calculated using signal intensity measurements before (SI pre) and after (SI post) each time point of injection according to the following formula: RSE $(\%)=100 \times[1-($ SI post in tumor/ SI post in muscle)/(SI pre in tumor/SI pre in muscle)].

\section{In vivo biocompatibility}

A total of 15 male Kunming mice (4 weeks old) were purchased from Shanghai SLAC Laboratory Animal Co., Ltd., randomly divided into three groups $(n=5$ for each group), and then intravenously administered with $200 \mu \mathrm{L}$ USPIO, Apt-USPIO (Fe concentration: $1 \mathrm{mg} / \mathrm{mL}$ ), and PBS as a control, respectively. In the following 1-month period, the body weight of the mice was measured every other day. On the 30th day postinjection, all the mice were sacrificed and vital organs (heart, liver, spleen, lung, and kidney) harvested. These organs were fixed in $10 \%$ formalin, paraffinembedded, and stained with hematoxylin and eosin (H\&E) to observe the possible histopathological differences.

\section{Statistical analysis}

The statistical analyses were performed with SPSS software package (v. 16.0.1; SPSS Inc., Chicago, IL, USA), and $p$-values $<0.05$ were considered statistically significant. Differences of the tumor signal intensities in each group before and 1, 2, and $4 \mathrm{~h}$ after injection were analyzed by paired $t$-test. Differences in RSE values between groups at different time points after injection were analyzed by independent $t$-test. One-way analysis of variance was performed to compare the difference in body weight among the three groups of the Kunming mice on the 30th day postinjection.

\section{Results}

\section{Preparation, characterization, and verification of Apt-USPIO nanoparticle}

The preparation of Apt-USPIO is schematically shown in Figure 1.

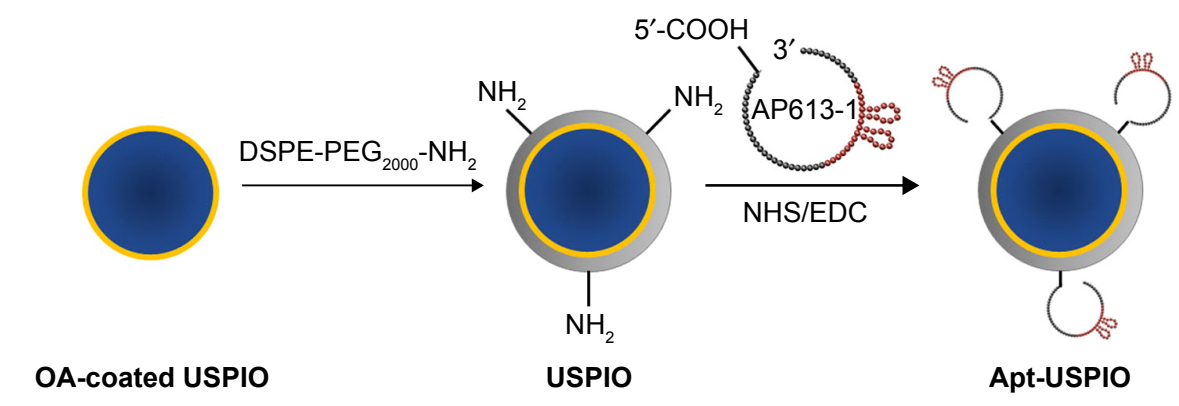

Figure I Schematic diagram of the synthesis of AP6I3-I-mediated USPIO (Apt-USPIO). OA-coated USPIO nanoparticles are modified with DSPE-PEG ${ }_{2000}$ NH $_{2}$. Amino moieties $\left(\mathrm{NH}_{2}-\right)$ of USPIO nanoparticles and 5' carboxyl (-COOH) of AP6I3-I are reacted to construct the Apt-USPIO probe.

Abbreviations: Apt-USPIO, aptamer-mediated USPIO; DSPE-PEG 2000 - $\mathrm{NH}_{2}$, I,2-distearoyl-sn-glycero-3-phosphoethanolamine-N-[amino(polyethylene glycol)-2000]; EDC, I-ethyl-3-(3-dimethylaminopropyl)carbodiimide hydrochloride; NHS, N-hydroxysuccinimide; OA, oleic acid; USPIO, ultrasmall superparamagnetic iron oxide. 
The average core size, morphology, and size distribution of Apt-USPIO were examined by TEM (Figure 2A and B). As shown in Figure 2A and B, the prepared nanoparticles were homogeneously dispersed without agglomeration, and round in shape with an average magnetic core size of approximately $10 \mathrm{~nm}$. After conjugation with AP613-1, the zeta potential decreased from $-17.9 \mathrm{mV}$ for USPIO to $-21.9 \mathrm{mV}$ for Apt-USPIO due to the presence of negative-charge aptamers on the surface of USPIO (Figure 2C). The hydrodynamic diameters of USPIO also increased from 38.0 to $45.2 \mathrm{~nm}$ after the formation of Apt-USPIO (Figure 2D). To evaluate the magnetic behavior of Apt-USPIO, the $\mathrm{T}_{1}$ and $\mathrm{T}_{2}$ relaxation times were measured. The corresponding longitudinal $\left(\mathrm{r}_{1}\right)$ and transverse $\left(\mathrm{r}_{2}\right)$ relaxivities were calculated as 14.3 and $50.2 \mathrm{mM}^{-1} \cdot \mathrm{s}^{-1}$ respectively, which demonstrated that Apt-USPIO could potentially be used as an MR $\mathrm{T}_{2}$ contrast agent.

Agarose gel electrophoresis showed different speeds of migration of Ap613-1, Apt-USPIO, and USPIO to the anode (Figure 3). The band of Apt-USPIO was shown to fall behind that of AP613-1. This indicated that the migration of AP613-1

A

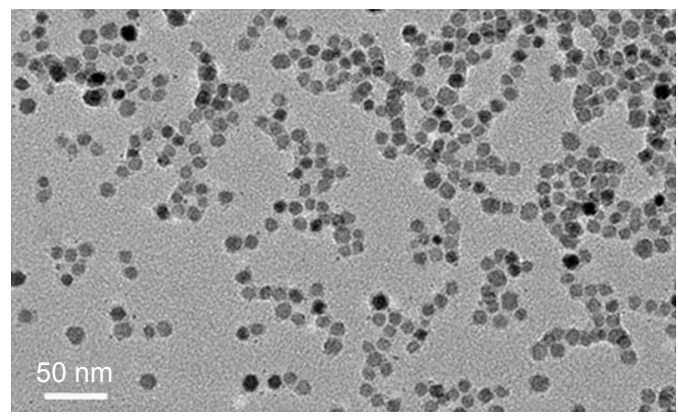

C

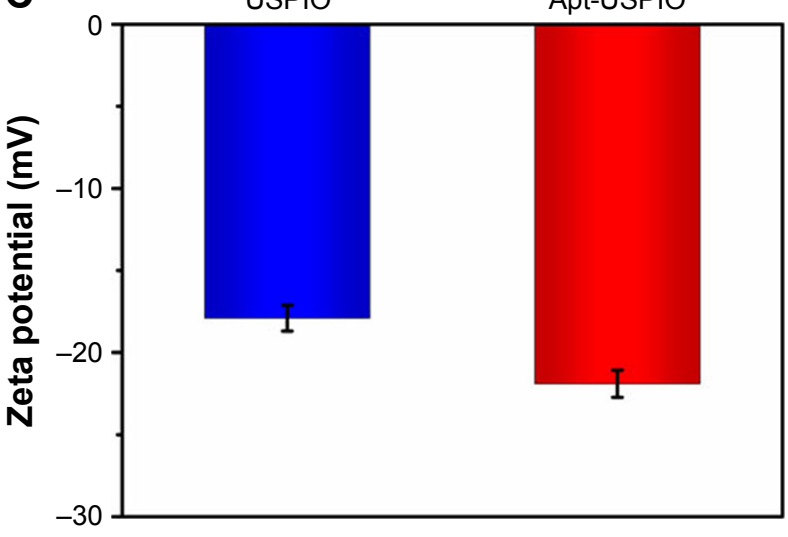

was hindered by USPIO because of the large particle size and weak negative charge. However, the band of Apt-USPIO preceded that of USPIO, which could be attributed to the existence of AP613-1 aptamer. The conjugation rate was estimated to be $80 \%$ after quantifying the unreacted aptamer in the supernatant. All these results confirm the successful construction of Apt-USPIO nanoparticles.

\section{Immunofluorescence staining of GPC3}

The anti-GPC3 antibody-based immunofluorescence assay (Figure 4) showed that the Huh-7 cells were positively stained while no specific staining was found in the normal L-02 cells, indicating strong GPC3 expression by Huh-7 cells.

\section{Cytotoxicity of nanoparticles}

CCK-8 test showed that the Huh-7 cells maintained $>94 \%$ cell viability even after incubation with USPIO and Apt-USPIO at the highest concentration of $200 \mu \mathrm{g} / \mathrm{mL}$ (Figure 5A). As the incubation time extended to $48 \mathrm{~h}$, over $90 \%$ cell viability was still observed at a concentration

B

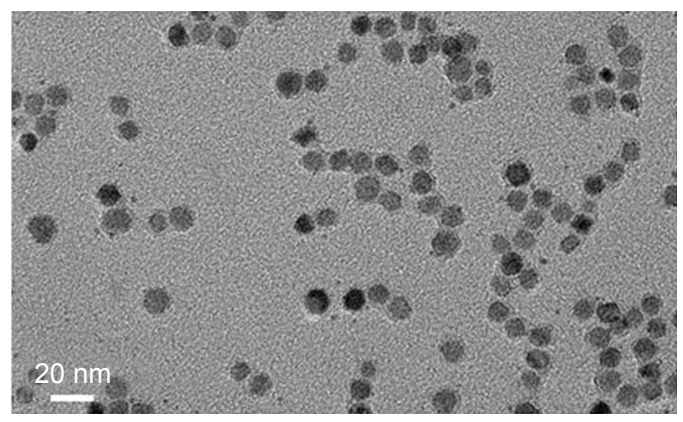

D

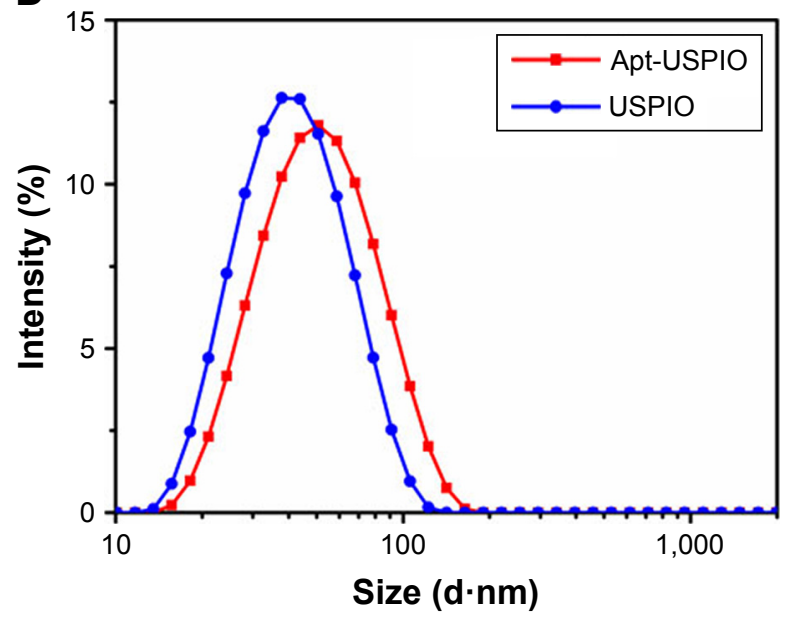

Figure 2 Characterization of Apt-USPIO and USPIO. (A and B) TEM images show that the Apt-USPIO nanoparticles disperse well, and are round in shape with an estimated magnetic core size of $10 \mathrm{~nm}$. (C) The zeta potential is $-17.9 \mathrm{mV}$ for USPIO and $-21.9 \mathrm{mV}$ for Apt-USPIO. (D) The hydrodynamic diameter is $38.0 \mathrm{~nm}$ for USPIO nm and $45.2 \mathrm{~nm}$ for Apt-USPIO.

Abbreviations: Apt-USPIO, aptamer-mediated USPIO; TEM, transmission electron microscopy; USPIO, ultrasmall superparamagnetic iron oxide. 


\section{A}

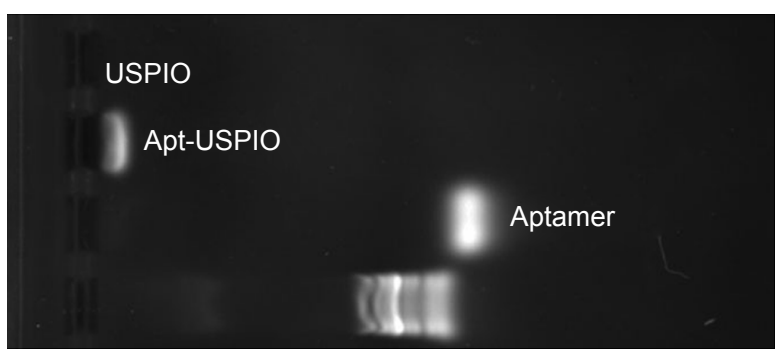

B

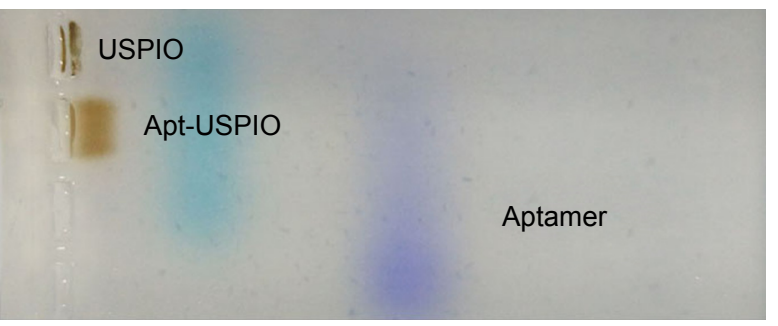

Figure 3 Electrophoresis tests. The (A) nucleic acid image and the (B) corresponding color photograph display different positions of aptamer (AP6I3-I), Apt-USPIO, and USPIO bands, representing their different speeds of migration to the anode with agarose gel electrophoresis.

Abbreviations: Apt-USPIO, aptamer-mediated USPIO; USPIO, ultrasmall superparamagnetic iron oxide.

of $200 \mu \mathrm{g} / \mathrm{mL}$ (Figure 5B). Similar results were observed when L-02 cells were exposed to USPIO and Apt-USPIO (Figure 5C and D). These findings demonstrated that there was very low cytotoxicity for both the tumor and normal cells when using USPIO and Apt-USPIO.

\section{Intracellular uptake experiments}

The specific uptake of Apt-USPIO by Huh-7 cells was verified by Prussian blue staining (Figure 6A-D). As opposed to the USPIO group, blue granules could be found in the cytoplasm of Huh-7 cells after $12 \mathrm{~h}$ incubation with Apt-USPIO, indicating ingestion of this probe. No specific staining was found in the L-02 cells incubated with either USPIO or Apt-USPIO.

\section{In vitro phantom MRI}

With the increase of the USPIO concentration, the signal intensity of Huh-7 cell suspension incubated with Apt-USPIO decreased progressively and more significantly than that with USPIO, and that of L-02 cells suspension incubated with USPIO and Apt-USPIO decreased as well (Figure 7A-D). This verified the preferential accumulation of Apt-USPIO in Huh-7 cells and subsequent lower signal on $\mathrm{T}_{2}$-weighted image caused by a higher concentration of $\mathrm{Fe}$ content.

\section{In vivo $M R I$ of xenograft models}

To confirm the ability of Apt-USPIO to target HCC in vivo, $M R \mathrm{~T}_{2}$-weighted imaging of Huh-7 xenografts was performed (Figure 8A), and the mean MR signal intensities of the tumors and the RSE values in Apt-USPIO and USPIO groups at different time points were listed (Figure 8B and C). Signal intensities of the tumors in the experimental group decreased significantly after intravenous injection of Apt-USPIO (preinjection vs 1, 2, and $4 \mathrm{~h}$ postinjection, $p<0.001$ ) (Figure 8D). Tumors showed the lowest signal intensity at $2 \mathrm{~h}$ after injection, and the signal intensity began to recover at $4 \mathrm{~h}$ after injection. However, for the tumors in the control mice, the signal intensities remained unchanged
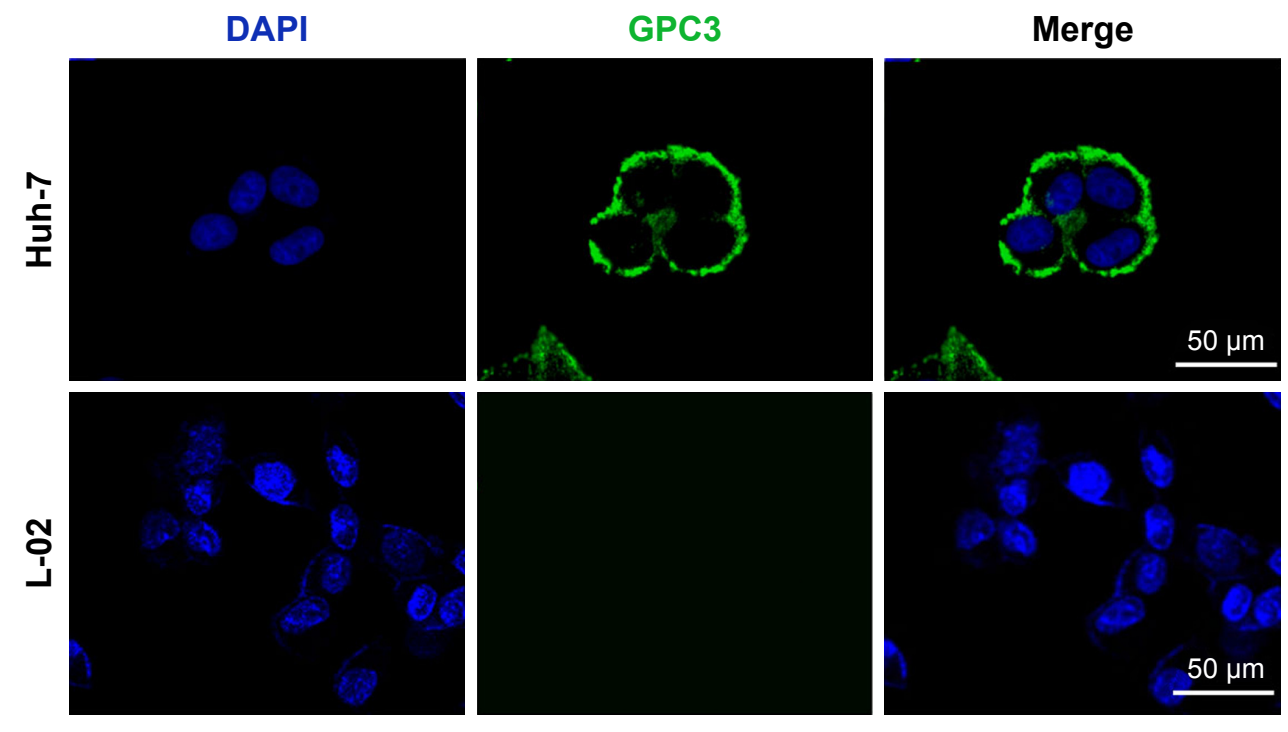

Figure 4 Immunofluorescence staining images of GPC3 show that Huh-7 cells are positively stained but L-02 cells are not. GPC3 represents the anti-GPC3 antibody binding (green) and DAPI locates the nuclei (blue).

Abbreviation: DAPI, 4',6-diamidino-2-phenylindole. 

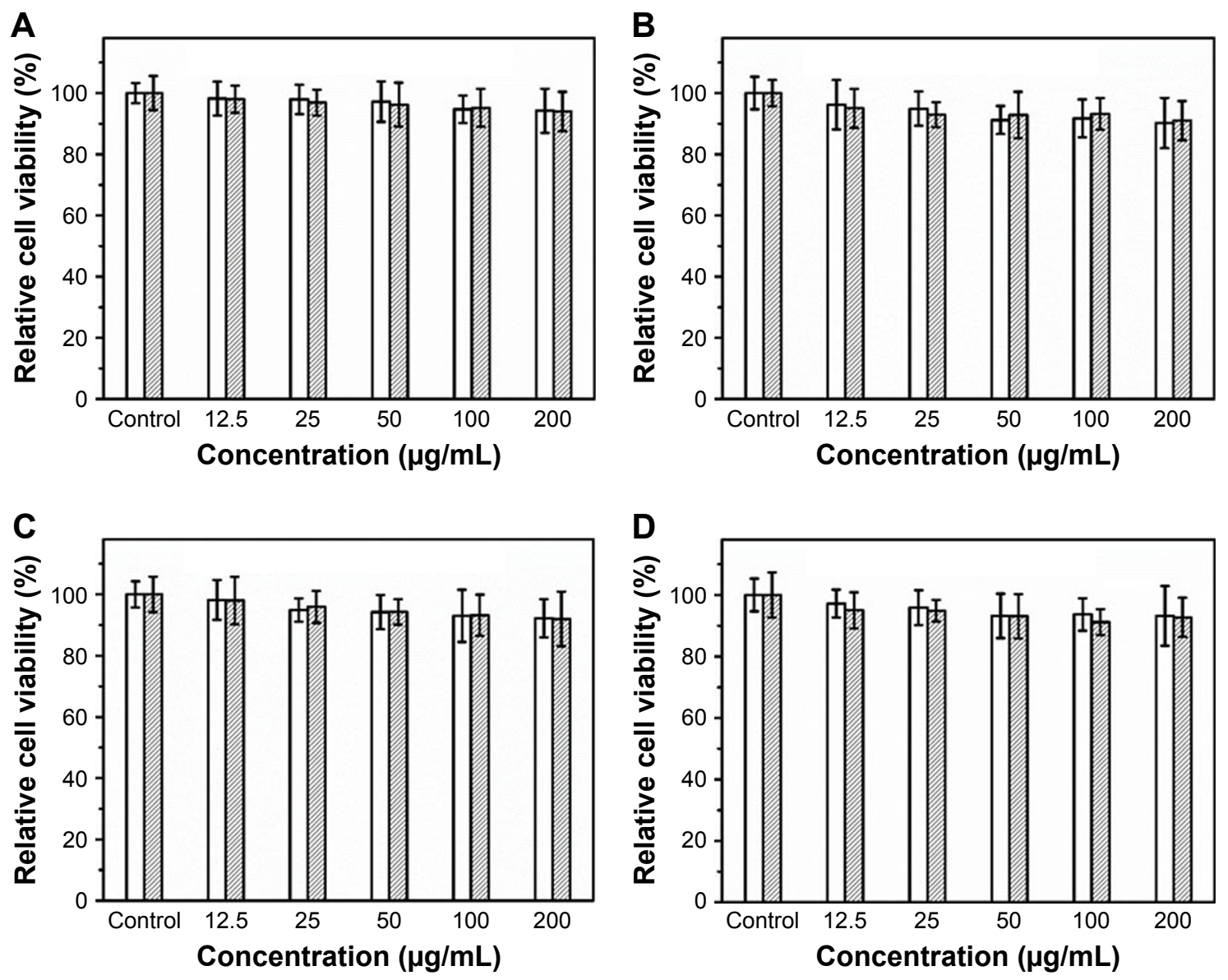

USPIO TIIISASPIO

Figure 5 Cytotoxicity of the USPIO and Apt-USPIO nanoparticles. In vitro viability study of Huh-7 cells in the presence of samples with varied concentrations for (A) 24 and (B) $48 \mathrm{~h}$, and that of L-02 cells for (C) 24 and (D) 48 h. There is no significant reduction of cell viability with the increased concentration of USPIO or Apt-USPIO and the prolonged incubation time.

Abbreviations: Apt-USPIO, aptamer-mediated USPIO; USPIO, ultrasmall superparamagnetic iron oxide.

before and after injection of the same amount of USPIO (preinjection vs $1 \mathrm{~h}$ postinjection, $p=0.237$; preinjection vs $2 \mathrm{~h}$ postinjection, $p=0.157$; preinjection vs $4 \mathrm{~h}$ postinjection, $p=0.260$ ) (Figure 8E). RSE values of the tumors in the Apt-USPIO group were significantly higher than tumors in the USPIO group at different time points $(1 \mathrm{~h}$ postinjection, $p<0.001 ; 2 \mathrm{~h}$ postinjection, $p<0.001 ; 4 \mathrm{~h}$ postinjection, $p=0.002$ ). Based on these results, specific binding of AptUSPIO to Huh-7 tumors in vivo was validated.

\section{In vivo biocompatibility}

After intravenous administration of USPIO, Apt-USPIO, and PBS for each group, all the Kunming mice were fed normally for 1 month. During this period, all the mice survived and behaved normally without loss of body weight (Figure 9A). On the 30th day, no significant difference in the body weight was found among the three groups $(p=0.606)$. The H\&E staining of the harvested organs (heart, liver, spleen, lung, and kidney) from the mice in the USPIO and Apt-USPIO groups showed no abnormality or acute inflammation in the tissues (Figure 9B), and these findings were identical to those from the control group administered with PBS. These results demonstrated the excellent in vivo biocompatibility of Apt-USPIO and USPIO.

\section{Discussion}

Different from traditional anatomical imaging, molecular imaging utilizes imaging probes to visualize target molecules of interest, and it plays a critical role in personalized oncology especially with recent advances in MR probes. ${ }^{21,22}$ MR probes are designed to allow targeted accumulation to highlight specific biomarkers with MRI, which can be accomplished through conjugation of targeting molecules with certain contrast agents. ${ }^{22}$ Biocompatible superparamagnetic iron 

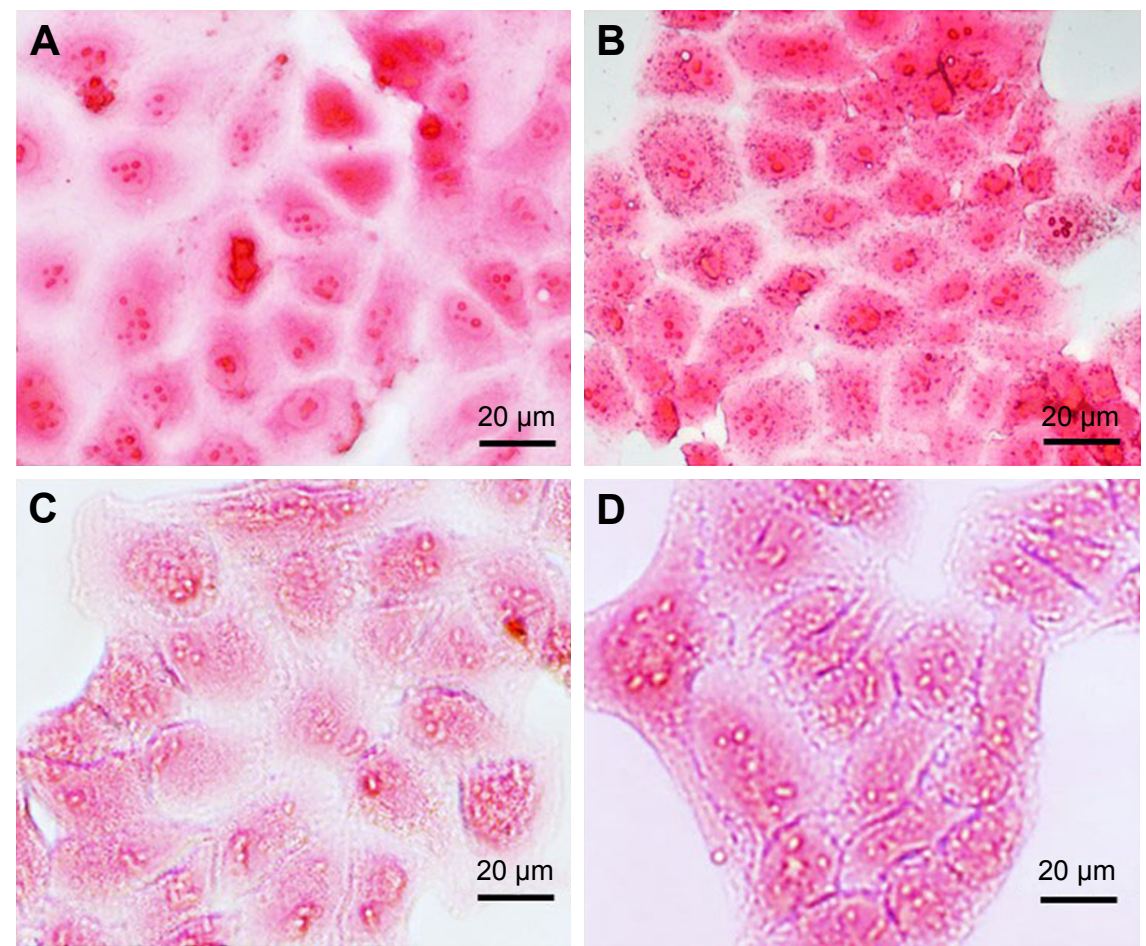

Figure 6 Intracellular uptake experiments. Prussian blue staining images of the Huh-7 cells after $12 \mathrm{~h}$ of incubation with (A) USPIO and (B) Apt-USPIO display the blue granules in the cytoplasm of Apt-USPIO-incubated Huh-7 cells, but no stain was found in the USPIO-incubated control cells. No specific stain was found in the L-02 cells incubated with (C) USPIO and (D) Apt-USPIO.

Abbreviations: Apt-USPIO, aptamer-mediated USPIO; USPIO, ultrasmall superparamagnetic iron oxide.

A

Huh-7

Apt-USPIO

USPIO
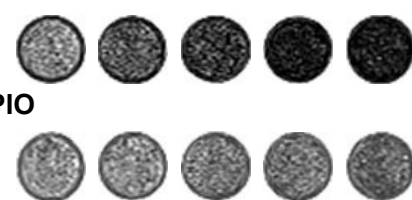

$\mu \mathrm{g} / \mathrm{mL} 0$

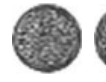

100150

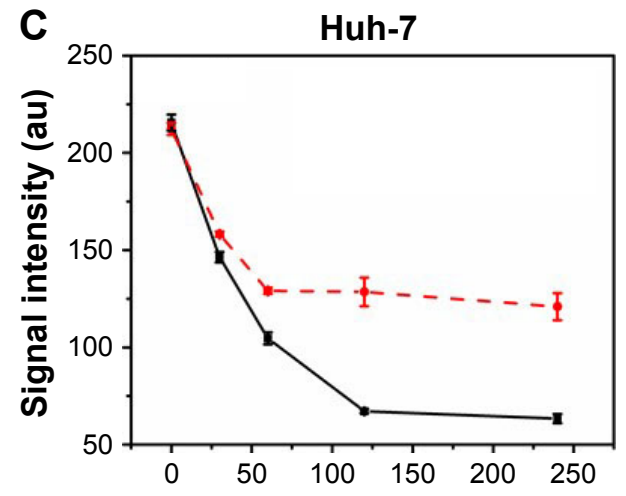

[Fe] concentration $(\mu \mathrm{g} / \mathrm{mL})$
B

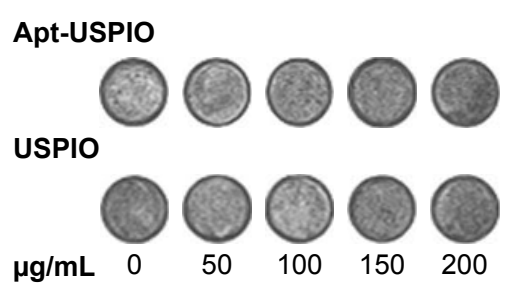

D

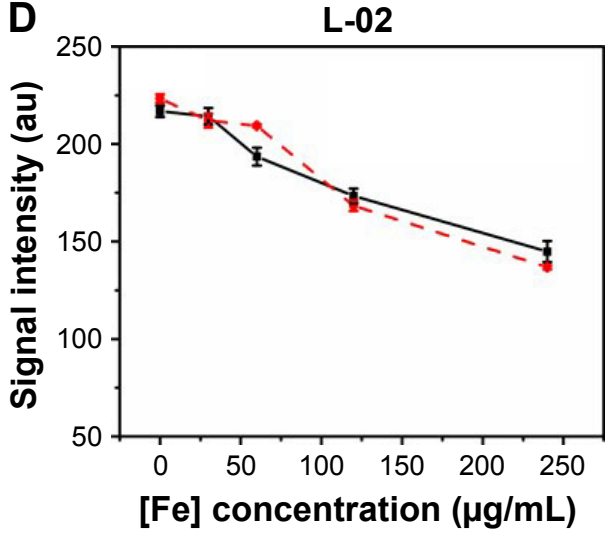

$$
\rightarrow-\text { Apt-USPIO }-\bullet-\text { USPIO }
$$

Figure 7 In vitro phantom MRI analysis. In vitro phantom $\mathrm{T}_{2}$-weighted images of Huh-7 cells $(\mathbf{A})$ and L-02 cells (B) incubated with various concentrations of Apt-USPIO and USPIO $(0,50,100,150$, and $200 \mu \mathrm{g} / \mathrm{mL})$ for $3 \mathrm{~h}$, and curves for the corresponding signal intensities of (C) Huh-7 and (D) L-02 cells show that the signal decline in the Huh-7 cells incubated with Apt-USPIO is much more pronounced than the other groups.

Abbreviations: Apt-USPIO, aptamer-mediated USPIO; MRI, magnetic resonance imaging; USPIO, ultrasmall superparamagnetic iron oxide. 

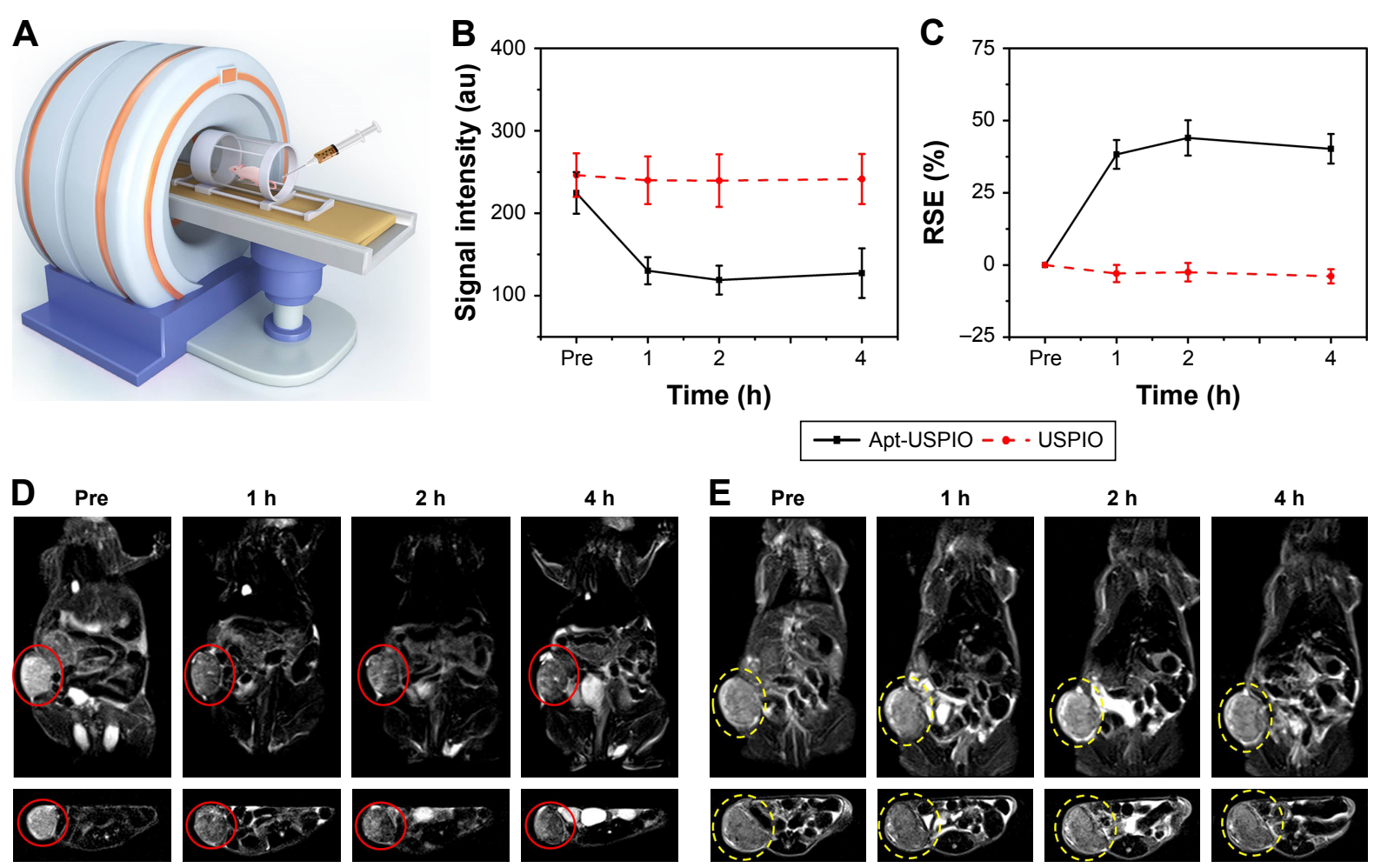

Apt-USPIO - - - USPIO

Figure 8 In vivo MRI of xenograft tumors. (A) In vivo $\mathrm{T}_{2}$-weighted MRI of the HCC xenograft models after injection of USPIO or Apt-USPIO. (B) $\mathrm{T}_{2}$-weighted MR signal intensities of the Huh-7 tumors significantly decrease in the experimental (Apt-USPIO) group as compared to the control (USPIO) group. (C) RSE values in the experimental group are significantly higher than the control group at different time points. (D) Coronal and axial $T_{2}$-weighted MR images of tumor-bearing mice before and after intravenous injection show significant negative enhancement of the tumors (red circles) at different time points in the Apt-USPIO group. (E) There is no decline in the signal intensities of the tumors (yellow circles) in the corresponding USPIO group.

Abbreviations: Apt-USPIO, aptamer-mediated USPIO; HCC, hepatocellular carcinoma; MRI, magnetic resonance imaging; RSE, relative signal enhancement; USPIO, ultrasmall superparamagnetic iron oxide.

oxide (SPIO) or USPIO nanoparticles with proper surface architecture attracted extensive research efforts for both targeted imaging and drug delivery applications after being functionalized with a series of biomolecules. ${ }^{23}$

With unique advantages, aptamers have been experimentally used as targeting ligands for the detection of various tumors including colorectal cancer, leukemia, glioma, breast, and prostate cancer., ${ }^{6,28-27}$ A prostate-specific membrane antigen aptamer-conjugated SPIO nanoparticle exhibiting preferential binding toward prostate cancer cells both in vitro and in vivo when analyzed by $\mathrm{T}_{2}$-weighted MRI has been reported..$^{18}$ Recently, an aptamer coupled with USPIO was fabricated to target vascular endothelial growth factor 165 overexpressed by some tumors. ${ }^{19}$ However, till now, reports on the application of aptamer-based MR probes specific for HCC are lacking. In this study, we successfully developed a novel HCC-targeted MR probe by conjugating GPC3-specific aptamer with USPIO. Apt-USPIO particles were found to be stable and biocompatible with very low cell toxicity. Both in vitro and in vivo experiments demonstrated preferential accumulation of Apt-USPIO probes in Huh-7 tumor cells.
To bind the GPC3-specific aptamer to the USPIO nanoparticles covalently, we cross-linked $5^{\prime}$ carboxyl-modified AP613-1 aptamer with amino PEG-modified USPIO by the formation of a stable peptide bond. Successful conjugation, which is a prerequisite for subsequent targeted imaging, was verified by a series of test findings including alterations in the hydrodynamic diameter and zeta potential, different migration speeds of AP613-1, Apt-USPIO, and USPIO, and reduced amounts of oligonucleotides in the supernatant. Furthermore, the conjugation yield was estimated as high as $80 \%$. The $\mathrm{r}_{2}$ relaxivity of Apt-USPIO was $50.2 \mathrm{mM}^{-1} \cdot \mathrm{s}^{-1}$, which indicated that it was suitable for use as a $T_{2}$ MRI contrast agent. ${ }^{16}$

It has been reported that GPC3 was highly expressed in the Huh-7 cell line, ${ }^{28}$ which was also confirmed by our immunofluorescence assay. Therefore, Huh- 7 cell line was chosen as the targeted HCC in the present study. Through our study, targeting capability of the Apt-USPIO to the Huh-7 cells was verified by the intracellular uptake experiments and in vitro phantom MRI, indicating that the binding activity of aptamer AP613-1 was not altered after a series of manipulations for the conjugation. 

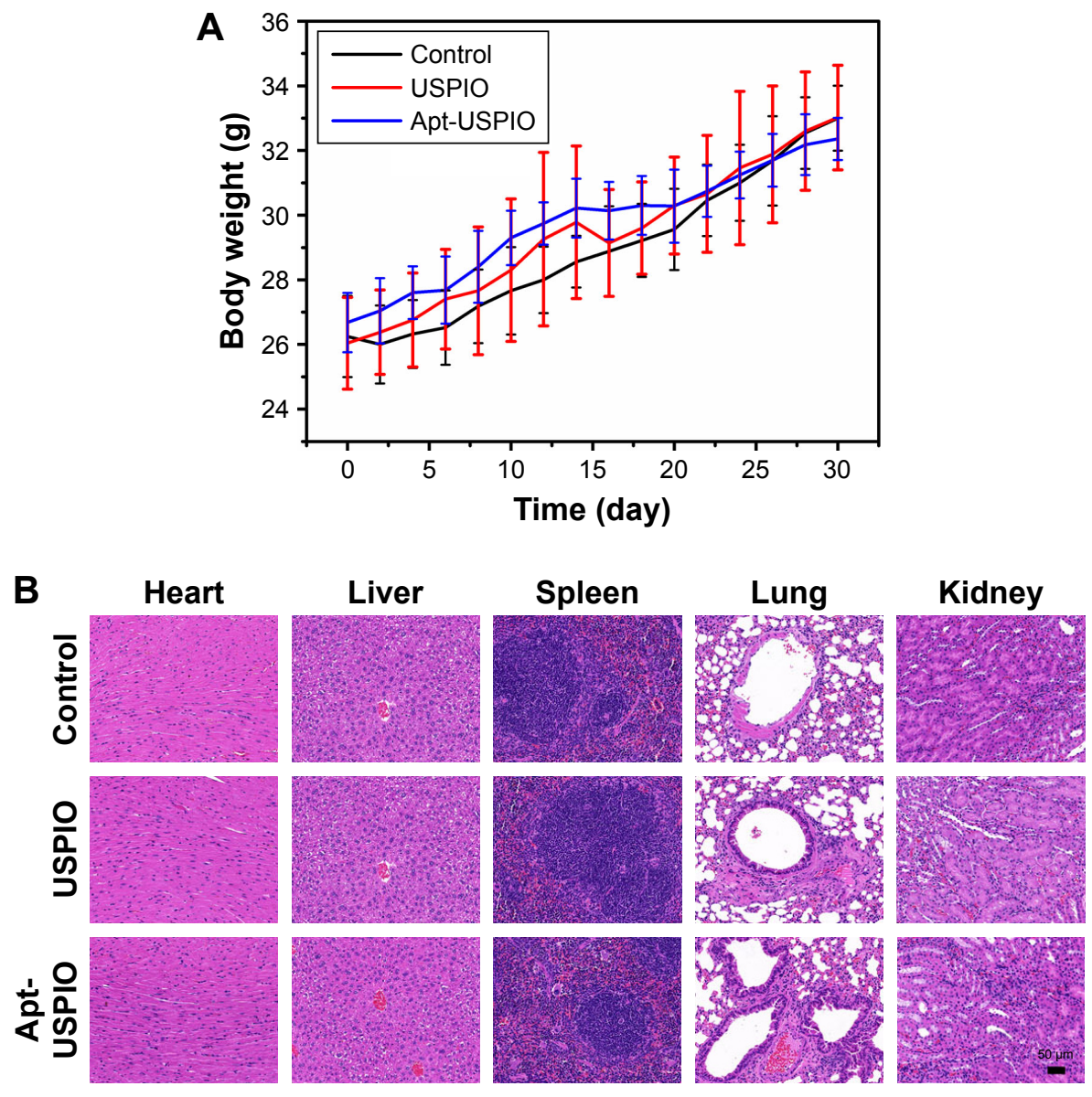

Figure 9 In vivo biocompatibility analyses. (A) After intravenous administration of USPIO, Apt-USPIO, and PBS (control), no significant difference in the body weight is found among the three groups of Kunming mice during the 30 days feeding. (B) H\&E staining of the vital organs including heart, liver, spleen, lung, and kidney from these mice in the three different groups shows normal findings.

Abbreviations: Apt-USPIO, aptamer-mediated USPIO; H\&E, hematoxylin and eosin; PBS, phosphate buffer solution; USPIO, ultrasmall superparamagnetic iron oxide.

Although target molecules in vivo are influenced by various physiological factors, and are not as stable and well defined as those in vitro, quite a few studies have successfully employed aptamers for in vivo-targeted imaging of xenograft tumors. ${ }^{6,18,19,26,29,30}$ In this study, excellent in vivo targeting was convincingly demonstrated by the significant negative enhancement of Huh-7 tumors on $\mathrm{T}_{2}$-weighted images after intravenous administration of Apt-USPIO, and the maximum negative enhancement was shown at $2 \mathrm{~h}$ after injection. Taking all these into consideration, we predict that aptamer-mediated molecular MRI could be extended to other tumors for earlier diagnosis and for assessing early response to treatment.

Unexpectedly, the signal intensity of the tumor remained unchanged on $\mathrm{T}_{2}$-weighted images after injection of USPIO alone. Similar phenomenon was reported by other authors. ${ }^{6,19,26}$ The possible contributing factors may include different acquisition times, blood supply of tumors, and different properties of nanoparticles.
Besides the targeting efficiency, biocompatibility of an imaging probe is another crucial issue for potential clinical application. PEG is a polymer usually used for surface modification of a magnetic core to improve colloidal stability and biocompatibility, and to enable functionalization with some targeting ligands. To facilitate conjugation with AP613-1 and further targeted imaging, USPIO nanoparticles were coated with PEG as in other studies. ${ }^{16,31}$ High biocompatibility of the Apt-USPIO was confirmed by the findings of CCK-8 assay and histopathology of vital organs in mice after its intravenous administration.

\section{Conclusion}

In summary, an MRI probe targeting $\mathrm{HCC}$ was fabricated by conjugating USPIO with an aptamer specific for GPC3. The stability, safety, and in vitro and in vivo GPC3-binding activity with $T_{2}$ negative enhancement characteristics in tumor xenografts suggested that this Apt-USPIO probe is a promising targeted MRI contrast agent for primary and 
metastatic HCC, which may be of great value and warrants further investigation.

\section{Disclosure}

The authors report no conflicts of interest in this work.

\section{References}

1. Torre LA, Bray F, Siegel RL, Ferlay J, Lortet-Tieulent J, Jemal A. Global cancer statistics, 2012. CA Cancer J Clin. 2015;65(2):87-108.

2. Venook AP, Papandreou C, Furuse J, de Guevara LL. The incidence and epidemiology of hepatocellular carcinoma: a global and regional perspective. Oncologist. 2010;15 Suppl 4:5-13.

3. Semelka RC, Helmberger TK. Contrast agents for MR imaging of the liver. Radiology. 2001;218(1):27-38.

4. Cruite I, Schroeder M, Merkle EM, Sirlin CB. Gadoxetate disodiumenhanced MRI of the liver: part 2, protocol optimization and lesion appearance in the cirrhotic liver. AJR Am J Roentgenol. 2010;195(1): 29-41.

5. Tan W, Donovan MJ, Jiang J. Aptamers from cell-based selection for bioanalytical applications. Chem Rev. 2013;113(4):2842-2862.

6. Kang WJ, Lee J, Lee YS, et al. Multimodal imaging probe for targeting cancer cells using uMUC-1 aptamer. Colloids Surf B Biointerfaces. 2015;136: 134-140.

7. Jin C, Zheng J, Li C, Qiu L, Zhang X, Tan W. Aptamers selected by cellSELEX for molecular imaging. J Mol Evol. 2015;81(5-6):162-171.

8. Libbrecht L, Severi T, Cassiman D, et al. Glypican-3 expression distinguishes small hepatocellular carcinomas from cirrhosis, dysplastic nodules, and focal nodular hyperplasia-like nodules. Am J Surg Pathol. 2006;30(11):1405-1411

9. Wang XY, Degos F, Dubois S, et al. Glypican-3 expression in hepatocellular tumors: diagnostic value for preneoplastic lesions and hepatocellular carcinomas. Hum Pathol. 2006;37(11):1435-1441.

10. Yamauchi N, Watanabe A, Hishinuma M, et al. The glypican 3 oncofetal protein is a promising diagnostic marker for hepatocellular carcinoma. Mod Pathol. 2005;18(12):1591-1598.

11. Haruyama Y, Kataoka H. Glypican-3 is a prognostic factor and an immunotherapeutic target in hepatocellular carcinoma. World $J$ Gastroenterol. 2016;22(1):275-283.

12. Hsu HC, Cheng W, Lai PL. Cloning and expression of a developmentally regulated transcript MXR7 in hepatocellular carcinoma: biological significance and temporospatial distribution. Cancer Res. 1997; 57(22):5179-5184.

13. Capurro M, Wanless IR, Sherman M, et al. Glypican-3: a novel serum and histochemical marker for hepatocellular carcinoma. Gastroenterology. 2003;125(1):89-97.

14. Zhao M, Dong L, Liu Z, Yang S, Wu W, Lin J. In vivo fluorescence imaging of hepatocellular carcinoma using a novel GPC3-specific aptamer probe. Quant Imaging Med Surg. 2018;8(2):151-160.

15. Di Marco M, Sadun C, Port M, Guilbert I, Couvreur P, Dubernet C. Physicochemical characterization of ultrasmall superparamagnetic iron oxide particles (USPIO) for biomedical application as MRI contrast agents. Int J Nanomedicine. 2007;2(4):609-622.
16. Lee N, Yoo D, Ling D, Cho MH, Hyeon T, Cheon J. Iron oxide based nanoparticles for multimodal imaging and magnetoresponsive therapy. Chem Rev. 2015;115(19):10637-10689.

17. Li Y, Chen Z, Li F, Wang J, Zhang Z. Preparation and in vitro studies of MRI-specific superparamagnetic iron oxide antiGPC3 probe for hepatocellular carcinoma. Int J Nanomedicine. 2012;7:4593-4611.

18. Yu MK, Kim D, Lee IH, So JS, Jeong YY, Jon S. Image-guided prostate cancer therapy using aptamer-functionalized thermally crosslinked superparamagnetic iron oxide nanoparticles. Small. 2011;7(15): 2241-2249.

19. You XG, Tu R, Peng ML, et al. Molecular magnetic resonance probe targeting VEGF165: preparation and in vitro and in vivo evaluation. Contrast Media Mol Imaging. 2014;9(5):349-354.

20. Zhang L, He R, Gu H. Oleic acid coating on the monodisperse magnetite nanoparticles. Appl Surf Sci. 2006;253(5):2611-2617.

21. McDermott S, Kilcoyne A. Molecular imaging - its current role in cancer. QJM. 2016;109(5):295-299.

22. McMahon MT, Chan KW. Developing MR probes for molecular imaging. Adv Cancer Res. 2014;124:297-327.

23. Wang YX, Idee JM. A comprehensive literatures update of clinical researches of superparamagnetic resonance iron oxide nanoparticles for magnetic resonance imaging. Quant Imaging Med Surg. 2017; $7(1): 88-122$.

24. Jacobson O, Weiss ID, Wang L, et al. 18F-labeled single-stranded DNA aptamer for PET imaging of protein tyrosine kinase-7 expression. J Nucl Med. 2015;56(11):1780-1785.

25. Shi H, He X, Wang K, et al. Activatable aptamer probe for contrastenhanced in vivo cancer imaging based on cell membrane proteintriggered conformation alteration. Proc Natl Acad Sci U S A. 2011; 108(10):3900-3905.

26. Hwang DW, Ko HY, Lee JH, et al. A nucleolin-targeted multimodal nanoparticle imaging probe for tracking cancer cells using an aptamer. J Nucl Med. 2010;51(1):98-105.

27. Roy K, Kanwar RK, Kanwar JR. LNA aptamer based multi-modal, $\mathrm{Fe}_{3} \mathrm{O}_{4}$-saturated lactoferrin $\left(\mathrm{Fe}_{3} \mathrm{O}_{4}\right.$-bLf) nanocarriers for triple positive (EpCAM, CD133, CD44) colon tumor targeting and NIR, MRI and CT imaging. Biomaterials. 2015;71:84-99.

28. Lei CJ, Yao C, Pan QY, et al. Lentivirus vectors construction of SiRNA targeting interference GPC3 gene and its biological effects on liver cancer cell lines Huh-7. Asian Pac J Trop Med. 2014;7(10):780-786.

29. Mosafer J, Abnous K, Tafaghodi M, Mokhtarzadeh A, Ramezani M In vitro and in vivo evaluation of anti-nucleolin-targeted magnetic PLGA nanoparticles loaded with doxorubicin as a theranostic agent for enhanced targeted cancer imaging and therapy. Eur J Pharm Biopharm. 2017;113:60-74.

30. Wei Z, Wu Y, Zhao Y, et al. Multifunctional nanoprobe for cancer cell targeting and simultaneous fluorescence/magnetic resonance imaging. Anal Chim Acta. 2016;938:156-164.

31. Wu L, Mendoza-Garcia A, Li Q, Sun S. Organic phase syntheses of magnetic nanoparticles and their applications. Chem Rev. 2016;116(18): 10473-10512.
International Journal of Nanomedicine

\section{Publish your work in this journal}

The International Journal of Nanomedicine is an international, peerreviewed journal focusing on the application of nanotechnology in diagnostics, therapeutics, and drug delivery systems throughout the biomedical field. This journal is indexed on PubMed Central, MedLine, CAS, SciSearch ${ }^{\circledR}$, Current Contents ${ }^{\circledR} /$ Clinical Medicine,

\section{Dovepress}

Journal Citation Reports/Science Edition, EMBase, Scopus and the Elsevier Bibliographic databases. The manuscript management system is completely online and includes a very quick and fair peer-review system, which is all easy to use. Visit http://www.dovepress.com/ testimonials.php to read real quotes from published authors. 